\title{
APS Linac Klystron and Accelerating Structure Gain Measurements and Klystron PFN Voltage Regulation Requirements
}

\author{
Nicholas S. Sereno
}

\begin{abstract}
This note details measurements of the APS positron linac klystron and accelerating structure gain and presents an analysis of the data using fits to simple mathematical models. The models are used to investigate the sensitivity of the energy dependence of the output positron beam to klystron parameters. The gain measurements are separated into two parts: first, the energy gains of the accelerating structures of the positron linac are measured as a function of output power of the klystron; second, the klystron output power is measured as a function of input drive power and pulse forming network (PFN) voltage. This note concentrates on the positron linac rf and its performance as it directly affects the energy stability of the positron beam injected into the positron accumulator ring (PAR). Ultimately it is important to be able to minimize beam energy variations to maximize the PAR accumulation efficiency.
\end{abstract}

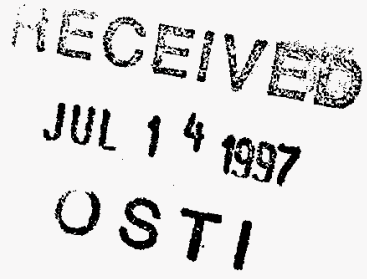

\section{Introduction}

The PAR can capture and accumulate a positron beam with a relative energy spread of $\pm 1 \%$ (1). It is therefore desirable to keep the relative centroid energy variation of the beam due to positron linac rf sources as small as possible. Typically one would like the maximum relative energy variation of the beam to be less than $\pm 0.1 \%$ so that, for a beam uniformly distributed in energy, the particles lost due to the PAR energy acceptance is $10 \%$. For more realistic energy distributions (such as a Gaussian) the fraction of particles lost due to the PAR energy acceptance is expected to be on the order of a few percent for $\pm 0.1 \%$ maximum centroid energy variation.

Figure 1 shows the positron linac which consists of three sectors of klystrons each powering a set of accelerating structures. The first linac sector (L3) downstream of the positron target consists of a single accelerating structure driven by a single klystron. The second and third linac sectors ( $L 4$ and L5) each consist of four accelerating structures driven by a klystron and a SLED (2). Output power variations in one or all of the positron linac klystrons result in energy variations in the positron beam injected into the PAR. Klystron power fluctuations are a direct result of variations in the klystron PFN voltage and/or input drive power.

In this report, measurements of the accelerating structure energy gain as a function of klystron (SLED) output power together with measurements of the klystron output power as a function of input drive power and PFN voltage are used to empirically determine the expected positron beam energy variation for

mETRIBUTION OF THIS DOCUMENT IS UNLIMTTED

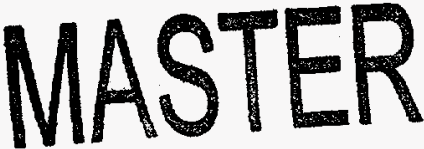




\section{DISCLAMERR}

Portions of this document may be illegible in electronic image products. Images are produced from the best available original document. 

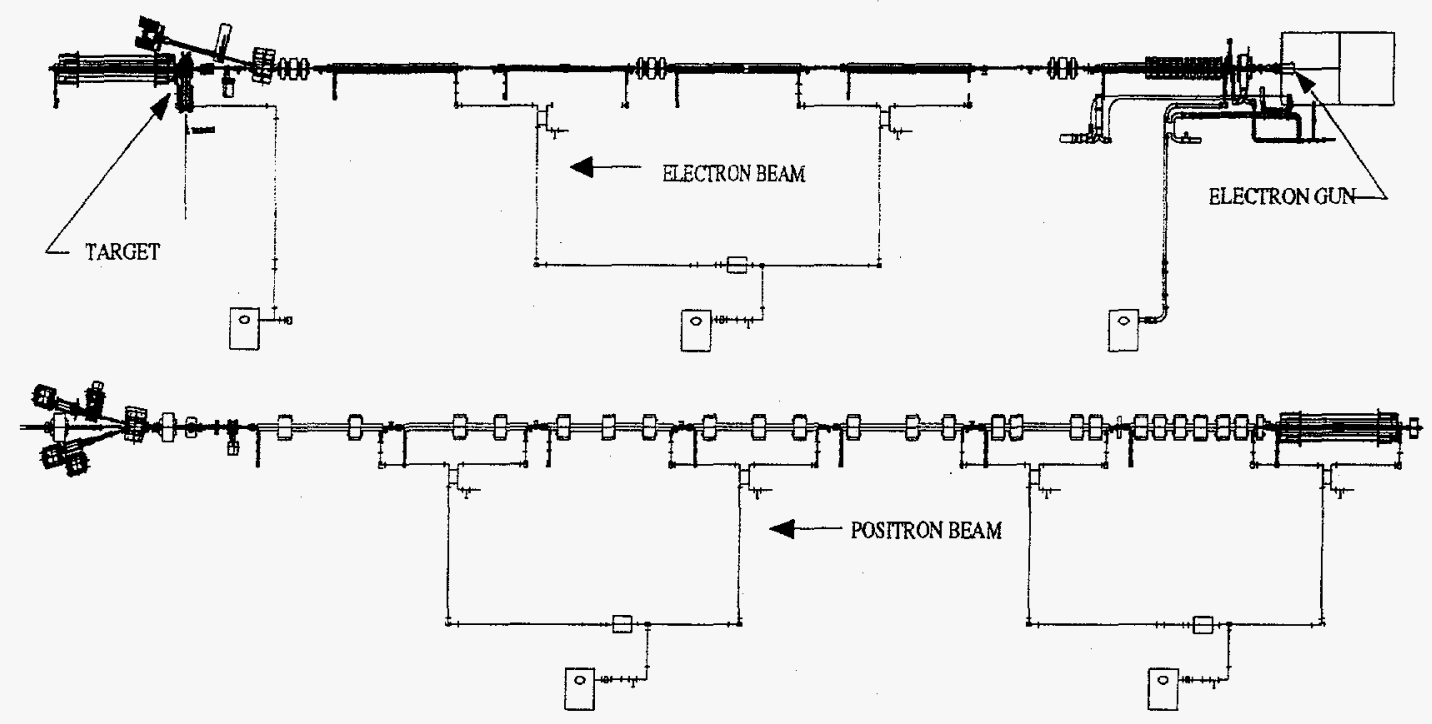

Figure 1. Layout of the APS linear accelerator showing the gun, accelerating structures, magnets, and target. The top part of the figure shows the electron linac layout from the $100-\mathrm{kV}$ gun to one accelerating structure past the target. The bottom part of the figure shows the positron linac layout from one accelerating structure past the target to the linac-to-PAR (LTP) transport line. Shown in the figure are nine accelerating structures connected to three klystrons in the positron linac.

given variations in the klystron drive power and PFN voltage. The data show that it is primarily variations in the PFN voltage that result in klystron output power fluctuations because the klystrons are typically run at saturation where large changes in klystron input drive power are required to make any significant change in the klystron output power. Hence, this report will be concerned with klystron output power stability as it relates to the PFN voltage. In addition, the phase of the output of each klystron was measured as a function of PFN voltage to determine how sensitive energy fluctuations are to PFN-induced phase errors. Finally, the report concludes with a simple model of klystron operation that describes the empirically derived results.

\section{Accelerating Structure Effective Shunt Impedance for Sectors L3, L4, and L5}

The measurements in this section quantify the energy gain of the linac accelerating structures as a function of klystron (or SLED) output power. The linac was set up to transport a low emittance, high energy ( $>200 \mathrm{MeV}$ ) electron beam to the linac-to-PAR (LTP) by removing the positron production target. The measurement was done by starting with all three sectors in the positron linac at full klystron output power (and hence the energy gain imparted to the beam was maximum) and varying the power in only one sector at a time. Starting each measurement with the klystron at full power was an attempt to minimize focusing and steering errors due to the (substantial) energy change of the beam as it passed through the quadrupoles. The incoming trajectory as 
Table 1. Summary of energy gain data taken for linac sectors L3, L4, and L5.

\begin{tabular}{|c||c|c|c|c|c|}
\hline \multirow{2}{*}{$\begin{array}{c}\text { Linac } \\
\text { Sector }\end{array}$} & Date & $\begin{array}{c}\text { Klystron } \\
\mathrm{R}(\mathrm{M} \Omega)\end{array}$ & $\begin{array}{c}\text { Klystron } \\
\text { Average } \\
\mathrm{R}(\mathrm{M} \Omega)\end{array}$ & $\begin{array}{c}\text { SLED } \\
\mathrm{R}(\mathrm{M} \Omega)\end{array}$ & $\begin{array}{c}\text { SLED } \\
\text { Average } \\
\mathrm{R}(\mathrm{M} \Omega)\end{array}$ \\
\hline \hline \multirow{3}{*}{ L3 } & $7-17-95$ & $139 \pm 1$ & & $\mathrm{NA}$ & \\
& $7-18-95$ & $153 \pm 1$ & & $\mathrm{NA}$ & \\
& $7-19-95$ & $134 \pm 1$ & & $\mathrm{NA}$ & \\
& $7-19-95$ & $133.1 \pm 0.6$ & 146 & $\mathrm{NA}$ & NA \\
& $7-22-95$ & $148.9 \pm 0.6$ & & $\mathrm{NA}$ & \\
& $7-22-95$ & $156.1 \pm 0.7$ & & $\mathrm{NA}$ & \\
& $7-22-95$ & $156 \pm 1$ & & $\mathrm{NA}$ & \\
\hline L4 & $7-17-95$ & $1228 \pm 15$ & 1221 & $420 \pm 6$ & 416 \\
& $7-18-95$ & $1214 \pm 3$ & & $412 \pm 1$ & \\
\hline \multirow{3}{*}{ L5 } & $5-29-95$ & $1450 \pm 10$ & & $278 \pm 2$ & \\
& $7-16-95$ & $1400 \pm 5$ & 1411 & $259.8 \pm 0.6$ & 266 \\
& $7-18-95$ & $1383 \pm 5$ & & $260.7 \pm 0.7$ & \\
\hline
\end{tabular}

well as spot profile at the LTP dipole are, however, observed to change as each sector is varied, with sectors L4 and L5 having the most effect because they impart the largest energy to the beam. An improvement to the measurement technique would be to use sddscontrollaw (3) to correct for trajectory errors as the beam energy is varied.

The energy gain of each linac sector can be parameterized by defining an effective shunt impedance according to

$$
E_{g}{ }^{2}=\mathrm{R} P
$$

where $E_{g}$ is the linac sector energy gain measured using the LTP dipole as a spectrometer (not shown in Figure 1), $P$ is the klystron or (SLED) forward power, and $\mathrm{R}$ is the shunt impedance. For sector L3, the shunt impedance is that of a single accelerating structure driven by a single klystron. For sectors L4 and L5 the impedance is that of four accelerating structures driven by a klystron and SLED combination. The output power of each klystron (or SLED) is measured via a coupler on the output accelerating structure. The energy gain for a given klystron is defined as the difference between the measured energy at a given output power and the energy at zero power. Figure 2 shows the data for sector L3 along with a linear fit after outlier data points two standard deviations away from the fit were eliminated (3). Data for the other sectors is similar and summarized in Table 1. The fit is constrained to go through zero energy gain at zero power. 


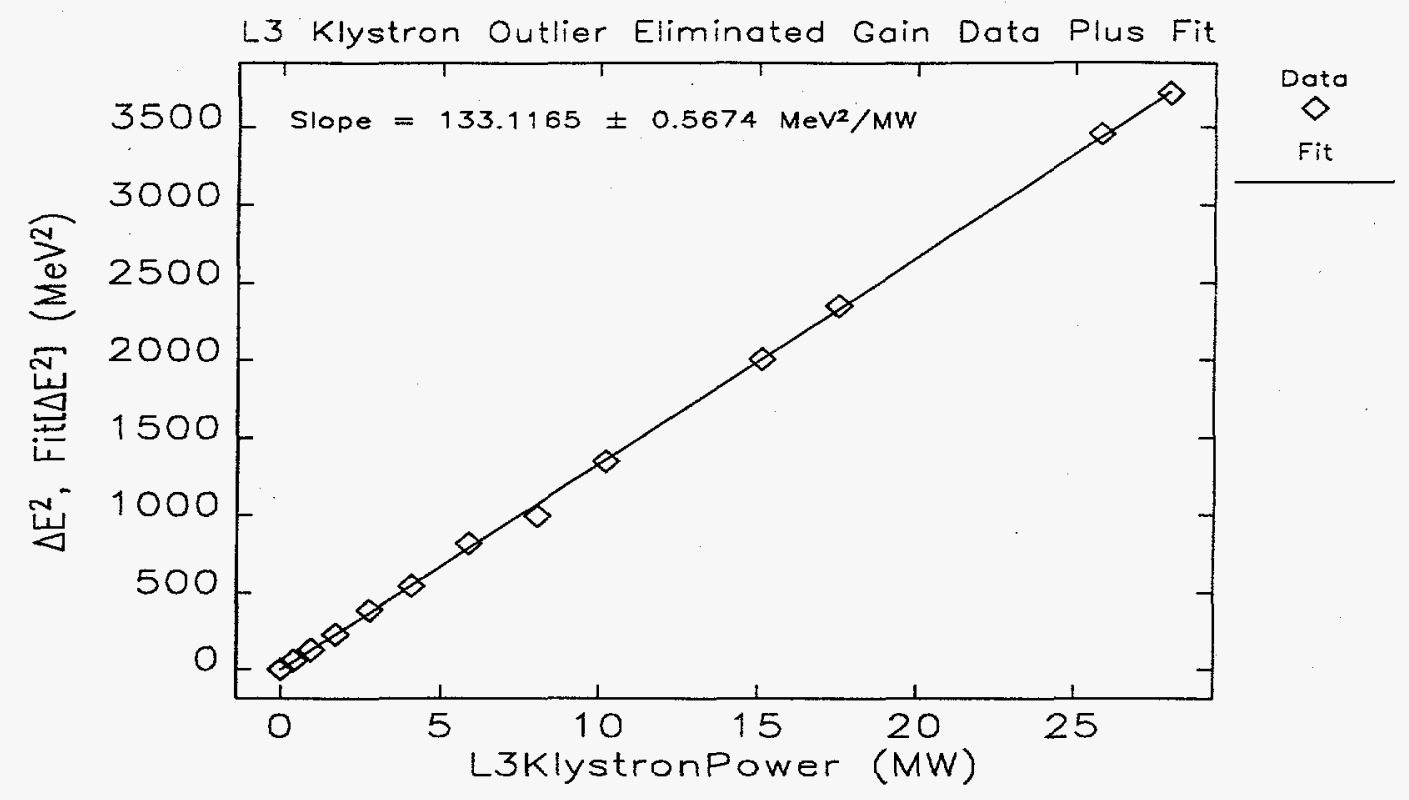

Figure 2. Sector L3 energy gain squared vs klystron output power. Shown is the slope of the line in $\mathrm{MeV}^{2} / \mathrm{MW}$ which are units equivalent to $\mathrm{M} \Omega$.

It is possible to explain the spread in the impedance values shown in the table by considering random energy measurement error. This error results from small changes in the trajectory before and after the LTP dipole (and hence bend angle) as the power in a specific linac sector klystron is varied. The error in the shunt impedance $R$ due to a trajectory error is estimated by referring to the equation of motion in a dipole given by

$$
\begin{aligned}
B \rho & =\frac{p}{e} \\
\rho & =\frac{l / 2}{\sin (\theta / 2)},
\end{aligned}
$$

where $\theta$ is the LTP dipole bend angle ( 0.2 radians) and $l$ is the effective length of the LTP dipole. Equation (3) is derived assuming the entrance and exit angles of the dipole are equal which is the case for the LTP dipole. The error in the energy determined by Eqs. (2) and (3) is due to the field error $\delta B$ and trajectory (angle) error $\delta \theta$. Standardization of the magnet was carried out for these measurements so the random field error is negligible. by

Considering only trajectory errors, the energy measurement error is given

$$
\frac{\delta p}{p}=\frac{1}{2} \cot (\theta / 2) \delta \theta .
$$


Given that the energy and momentum are numerically (nearly) identical for relativistic particles, Eq. (4) gives the relative energy error $(\delta E / E)$ for a trajectory error. Since the energy gain in Eq. (1) is the difference between the energy at any given power and the beam energy at zero power, the error in the energy gain is

$$
\frac{\delta E_{g}}{E_{g}}=\sqrt{2} \frac{\delta E}{E} .
$$

Using Eqs. (5), (4), and (1) the error in the shunt impedance is given in terms of the trajectory error as,

$$
\frac{\delta \mathrm{R}}{\mathrm{R}}=\sqrt{2} \cot (\theta / 2) \delta \theta
$$

Equation (6) indicates that a 1-mrad angle error caused by a trajectory offset results in a $1.4 \%$ shunt impedance error for a nominal LTP dipole bend angle of 0.2 radians. This amount of error is consistent with the variation in the impedance values from measurement to measurement except for sector L3. For L3, the spread in the impedance values is consistent with 5-mrad trajectory errors. Ultimately, other sources of errors could contribute significantly in this measurement. The trajectory error explanation just given is one plausible explanation of the data (except for an apparent outlier which corresponds to an unreasonably large $\sim 5$-mrad trajectory error). Other sources of error such as random power measurement error could be significant in the impedance measurement.

Sectors L4 and L5 show drastic differences in the effective impedance which are due to systematic calibration differences in the power measurement couplers, power meters, etc. in addition to real performance differences between the two systems. In particular, differences in SLED tuning between the two sectors is a possible explanation of the differences in the impedance data for these sectors.

\section{Klystron Gain Measurement for Sectors L3, L4, and L5}

In this measurement the klystron output power for each linac sector in the positron linac was measured as a function of input drive power and PFN voltage. The PFN voltage is applied to a 15:1 step up transformer that is connected to each klystron tube. Since the tube voltage and PFN voltage differ by a scale factor, the following analysis considers only the PFN voltage. An empirical formula was deduced from the data relating the output power to the drive power and PFN voltage. The sddstoolkit program sddsexperiment (3) was used to collect the data and vary the drive power and PFN voltage. The measured PFN voltage was averaged to reduce the effect of noise in the PFN voltage measurement circuit.

Data analysis consists of fitting an appropriate function to the measured output power data for a given PFN voltage. The chosen fitting function,

$$
P=a_{1}+a_{2} e^{a_{3} P_{i n}}
$$




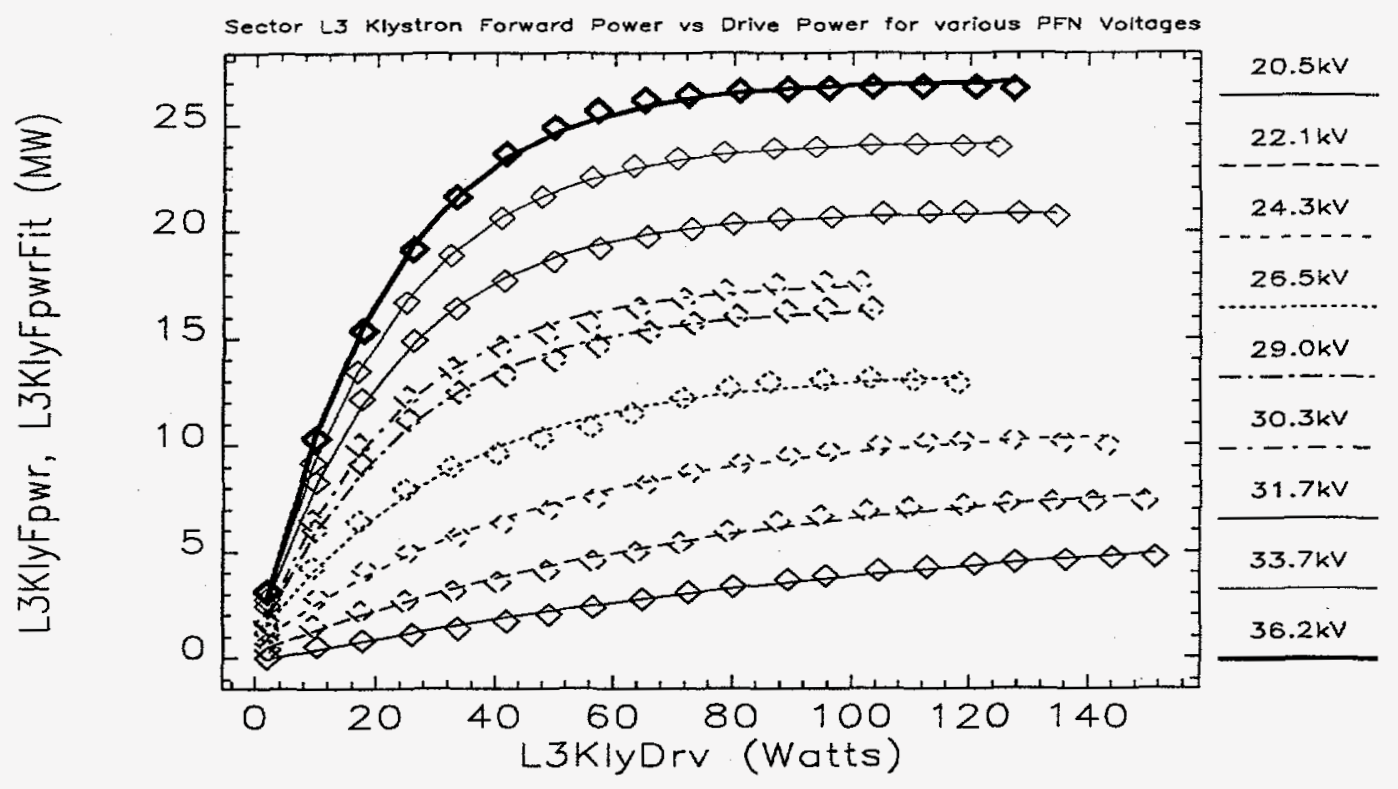

Figure 3. Sector L3 klystron output power vs input drive power for various PFN voltages.

reproduces the variation of output power with input power at a given PFN voltage adequately. For each PFN voltage, the fit will be slightly different and the fit coefficients in Eq. (7) can be considered functions of the PFN voltage. Each coefficient is then fit according to a simple linear function with a slope and intercept. In this way, Eq. (7) yields an empirical formula for the klystron output power in terms of both input power $\left(P_{i n}\right)$ and PFN voltage.

Figure 3 shows the output power of the sector L3 klystron as a function of drive power for various PFN voltages. Figures 4, 5, and 6 show the fit coefficients of $\mathrm{Eq}$. (7) as functions of the PFN voltage along with the linear fit. The data for sectors L4 and L5 show similar behavior. The data show that while the $a_{1}$ and $a_{2}$ coefficients are well described by a linear fit, the coefficient $a_{3}$ is not as well described by a linear fit as the other two, especially for PFN voltages near $30 \mathrm{kV}$. Table 2 summarizes the data for all three sectors and lists the slope and intercept of the linear fit to PFN voltage for each of the fit coefficients in Eq. ( 7 ).

At a given PFN voltage, outlier data beyond two standard deviations were eliminated in addition to data for input drive power values greater than the peak output power (saturation at a specific PFN voltage). Data beyond saturation was eliminated because the fitting function given by Eq. (7) cannot take into account the decrease of output power beyond saturation. This approximation is not too severe because the klystron is not normally run beyond 


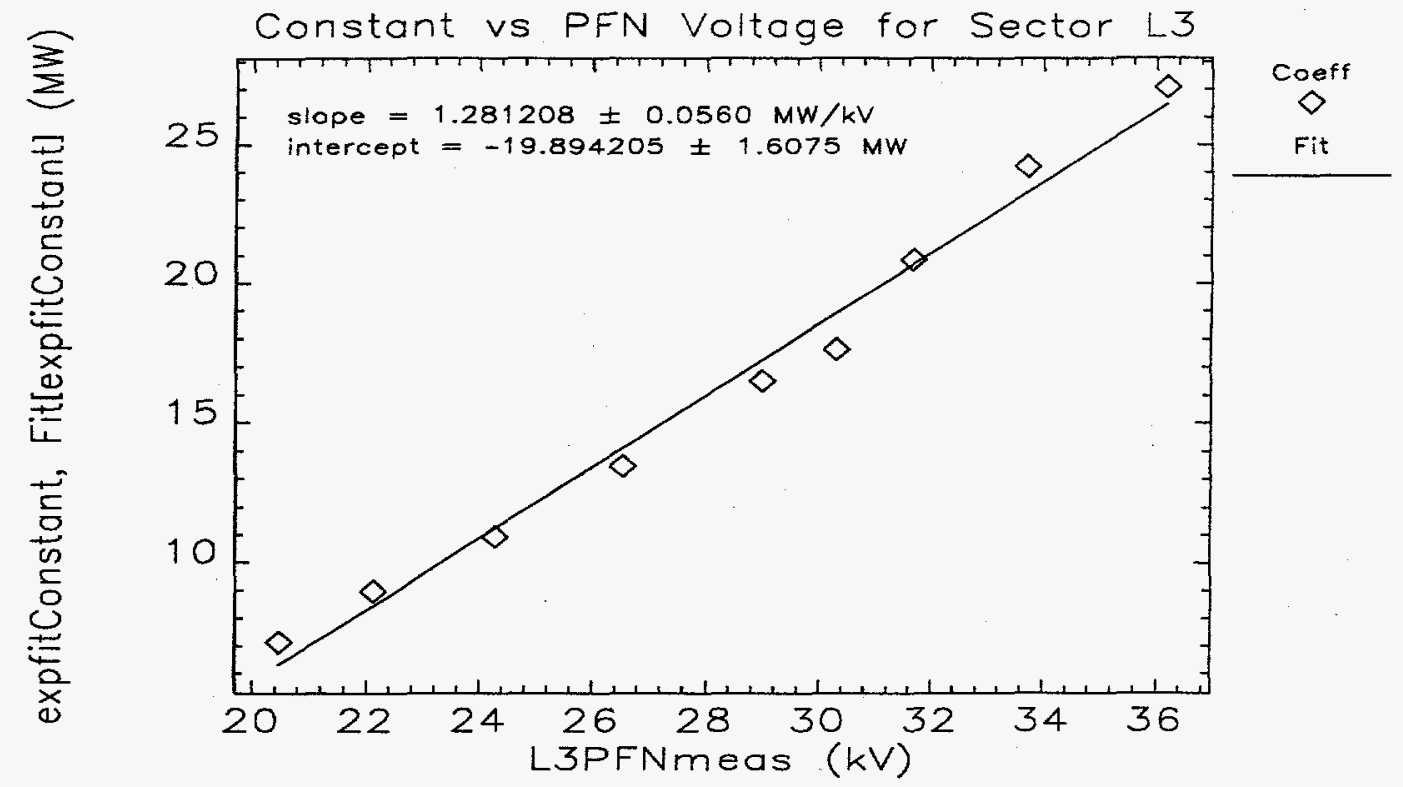

Figure 4. Coefficient $a_{1}$ as a function of PFN voltage along with a linear fit to the data.

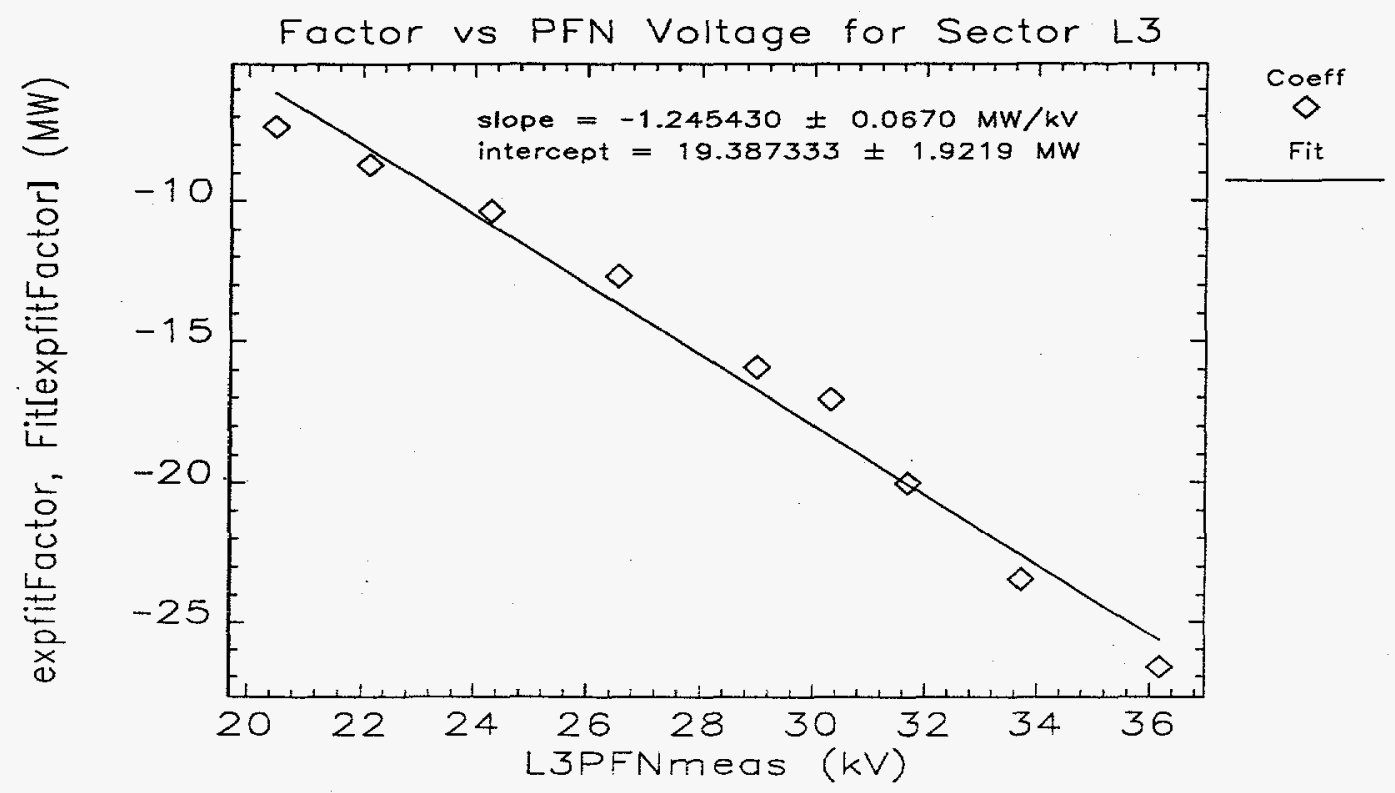

Figure 5. Coefficient $a_{2}$ as a function of PFN voltage along with a linear fit to the data. 


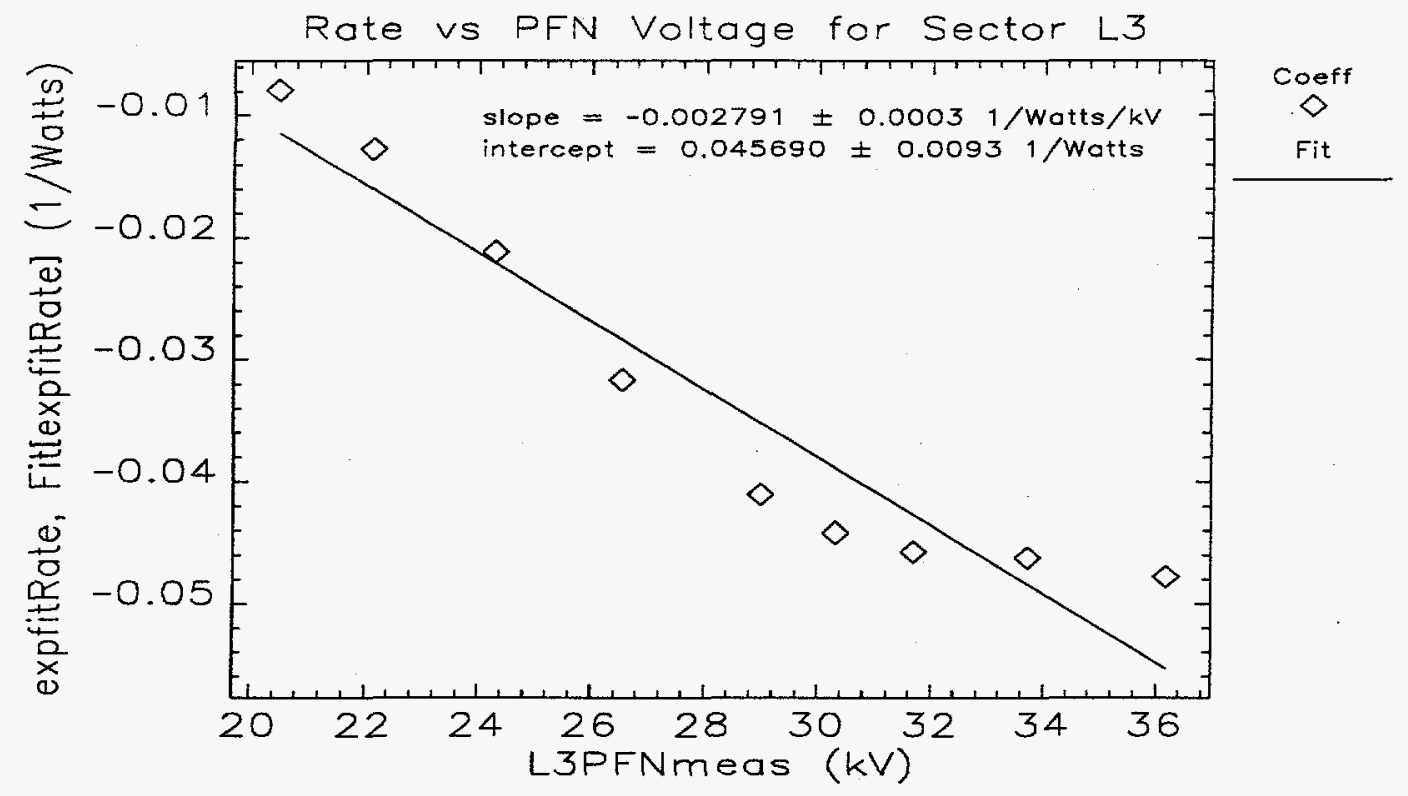

Figure 6. Coefficient $a_{3}$ as a function of PFN voltage along with a linear fit to the data.

saturation. To properly account for saturation effects, a more sophisticated model of the klystron is required.

Data taken for sectors L3 and L5 from 7-19-95 are listed in Table 2. Sector L4 data taken on the same date was of poor quality because of the relatively low PFN voltages that could be obtained before excessive vacuum problems occurred. Data taken on 1-15-95 for sector L4 are listed instead because they are of much better quality and were taken when this sector was delivering essentially the same performance as sector L5.

\section{Empirical Determination of the Allowable Modulator PFN Voltage Variation for Sectors L3, L4, and L5}

The empirical relations from the last two sections can now be used to quantify how much the PFN voltage of the positron linac modulators may be allowed to vary and still achieve a centroid energy stability of $< \pm 0.1 \%$. A significant source of PFN voltage variation is due to the booster ramped power supplies which lower the voltage of the $\mathrm{AC}$ line at a $2-\mathrm{Hz}$ rate. The presently installed PFN charging power supplies are not able to eliminate the $2-\mathrm{Hz}$ "ripple" of their output (PFN) voltage. The PFN voltage variation causes the klystron output power of each linac sector to vary at a $2-\mathrm{Hz}$ rate. The magnitude of the relative beam energy variation at injection into the PAR due to booster ramping is estimated to be about $\pm 0.5-1.0 \%$ or about the same order of 
Table 2. Summary of klystron gain coefficient linear fits for linac sectors L3, L4, and L5.

\begin{tabular}{|c||c|c|c|c|}
\hline $\begin{array}{c}\text { Linac } \\
\text { Sector }\end{array}$ & Date & Coefficient & Slope & Intercept \\
& & & & \\
\hline \hline \multirow{2}{*}{ L3 } & $7-19-95$ & $a_{1}$ & $1.26(\mathrm{MW} / \mathrm{kV})$ & $-20(\mathrm{MW})$ \\
& $7-19-95$ & $a_{2}$ & $-1.25(\mathrm{MW} / \mathrm{kV})$ & $19(\mathrm{MW})$ \\
& $7-19-95$ & $a_{3}$ & $-0.0028(1 /(\mathrm{kV} * W a t t s))$ & $0.046(1 /$ Watts $)$ \\
\hline \multirow{3}{*}{ L4 } & $1-15-95$ & $a_{1}$ & $1.47(\mathrm{MW} / \mathrm{kV})$ & $-19(\mathrm{MW})$ \\
& $1-15-95$ & $a_{2}$ & $-1.53(\mathrm{MW} / \mathrm{kV})$ & $21(\mathrm{MW})$ \\
& $1-15-95$ & $a_{3}$ & $-0.0031(1 /(\mathrm{kV} * W a t t s))$ & $0.054(1 / \mathrm{Watts})$ \\
\hline \multirow{2}{*}{ L5 } & $7-19-95$ & $a_{1}$ & $1.11(\mathrm{MW} / \mathrm{kV})$ & $-17(\mathrm{MW})$ \\
& $7-19-95$ & $a_{2}$ & $-1.13(\mathrm{MW} / \mathrm{kV})$ & $17.8(\mathrm{MW})$ \\
& $7-19-95$ & $a_{3}$ & $-0.0072(1 /(\mathrm{kV} * W a t t s))$ & $0.133(1 /$ Watts $)$ \\
\hline
\end{tabular}

magnitude as the PAR energy acceptance. In the following analysis, the PAR energy stability requirement is used along with the empirical relations derived previously to obtain an upper bound on the allowable PFN voltage variation.

The positron beam energy at the PAR is determined by adding up the maximum energy gain of sectors L3, L4, and L5

$$
E=E_{\circ}+E_{g}^{L 3}+E_{g}^{L 4}+E_{g}^{L 5}
$$

where $E_{0}$ is the energy of the captured beam after the target $(\sim 8 \mathrm{MeV})$ and the other terms are the energy gain for each positron linac sector. Combining Eqs. (1) and (7) for each sector and inserting them in Eq. (8) yields an equation for the energy of the positron beam injected into the PAR in terms of the PFN voltage of each sector.

A numerical calculation was done where the PFN voltage was varied about its nominal point for various final energy beams. The relative PFN voltage variation was found which resulted in a maximum relative beam energy variation of $\pm 0.1 \%$. The results are summarized in Table 3 . There are two assumptions under which these calculations were made. The first is that the klystrons are run at an input drive power that corresponds to saturation so as to maximize the output power for a given PFN voltage. This power is seen to be 100 Watts for sector L3 from Figure 2. Similarly for sectors L4 and L5 saturation occurs at an input drive power of 100 and 50 Watts, respectively. The second assumption is that each modulator is run at the same PFN voltage to produce a beam at the end of the linac with a given centroid energy. This second assumption is generally not how the modulator systems are run but is considered a good 
Table 3. PFN voltage variation required for a $\pm 0.1 \%$ beam energy variation.

\begin{tabular}{|c||c|c|}
\hline Centroid Energy (MeV) & PFN Voltage (kV) & PFN Voltage Variation \% \\
\hline \hline 273.4 & 23.5 & 0.05 \\
\hline 303.6 & 25.0 & 0.06 \\
\hline 338.4 & 27.0 & 0.08 \\
\hline 368.3 & 29.0 & 0.09 \\
\hline 394.7 & 31.0 & 0.10 \\
\hline 418.7 & 33.0 & 0.11 \\
\hline 441.0 & 35.0 & 0.12 \\
\hline 462.0 & 37.0 & 0.12 \\
\hline
\end{tabular}

approximation to actual operating conditions because the actual PFN voltages of each sector are typically within a few $\mathrm{kV}$ of one another.

The data in the table show empirically that

$$
\frac{\delta E}{E} \sim \frac{\delta V}{V},
$$

which is good to about $20 \%$ for energies above $\sim 338 \mathrm{MeV}$ which is the energy above which the PAR is able to accumulate and damp positrons efficiently. Saturation of the allowable PFN variation at high beam energies is due to the fact that the model used to represent the klystron output power given by Eq. (7) along with a linear fit of the coefficients of the model to the PFN voltage is only an approximation. Finally, the main empirical result from the data says that to obtain a relative energy variation of the beam into the PAR of a given percentage, the relative PFN voltage variation must be of the same order of magnitude.

\section{Empirical Determination of the Allowable Modulator PFN Voltage Variation for Sectors L3, L4, and L5 Assuming the Positron Beam Energy Variation is Due to Phase Variations}

In this section, the positron beam energy sensitivity to PFN-voltage-induced phase fluctuations is considered. The output phase of each positron linac klystron was measured as a function of PFN voltage for an input drive power corresponding to saturation as in the previous section. The data shown in Figure 7 are for sector L3. Table 4 summarizes the slope of the data for all three sectors in the positron linac. The average of the slopes listed in the table is about $17.8^{\circ} / \mathrm{kV}$. 


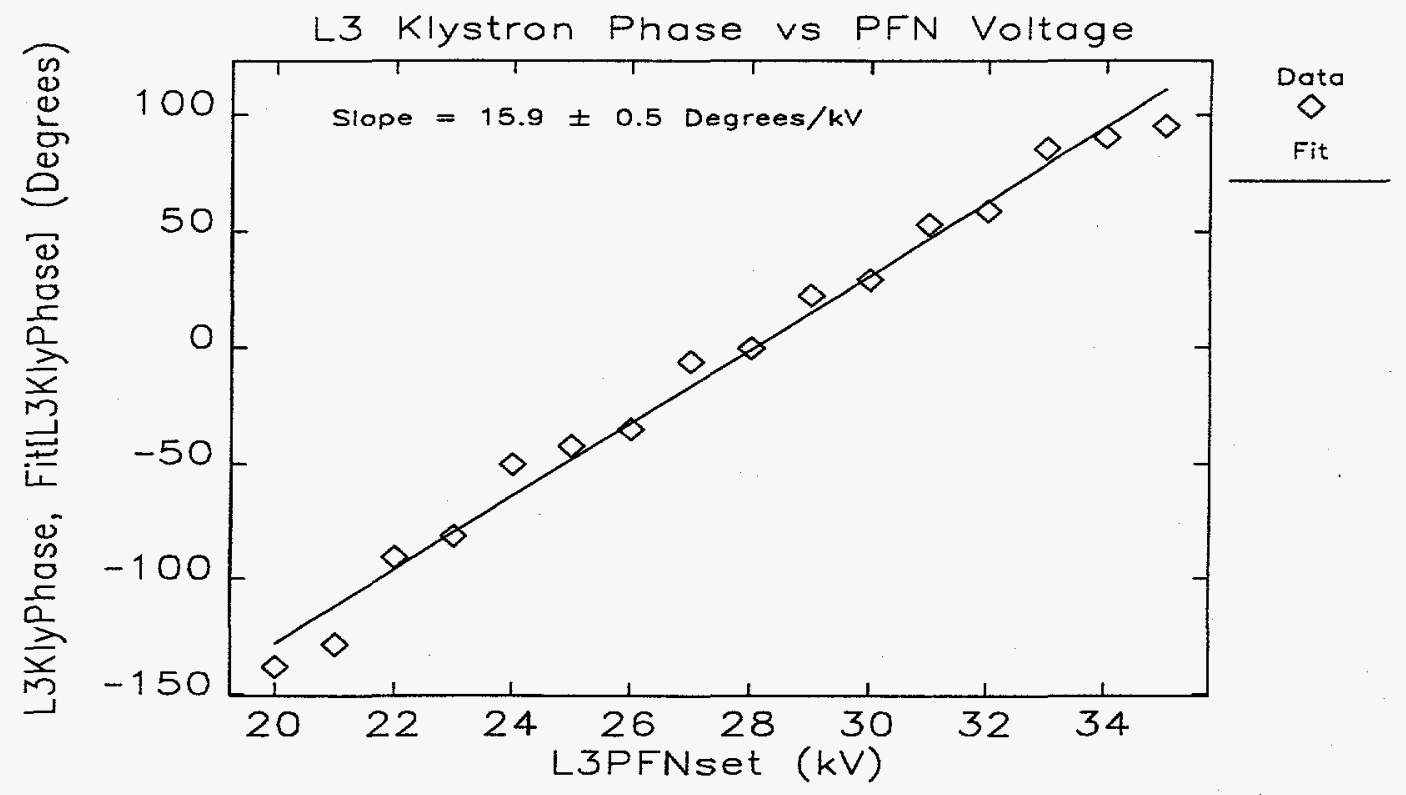

Figure 7. Sector L3 phase variation as a function of PFN voltage at saturation.

Table 4. Slope of fit for klystron and SLED phase vs PFN voltage data.

\begin{tabular}{|c||c|c|}
\hline Linac Sector & Klystron Slope $(\% / \mathrm{kV})$ & SLED Slope $(\% / \mathrm{kV})$ \\
\hline \hline L3 & 15.9 & NA \\
\hline L4 & 24.3 & 19.5 \\
\hline L5 & 14.3 & 15.1 \\
\hline
\end{tabular}


Using Eq. (7), the relative beam energy variation as a function of phase is given by

$$
\frac{\delta E}{E}=\left(\frac{E-E_{\circ}}{E}\right) \frac{\delta \phi^{2}}{2}
$$

assuming the beam passes through each linac sector on crest. The first factor in parentheses is essentially unity due to the fact that the initial energy $E_{\mathrm{o}} \sim$ $8 \mathrm{MeV}$ and the total energy $E \sim 350 \mathrm{MeV}$ during typical linac and PAR operation. Using Eq. (10), the maximum allowable phase variation for $\delta E / E \leq$ 0.001 is $2.6^{\circ}$. Using the average slope determined from Table 4 , this amount of phase variation corresponds to $\delta V=0.147 \mathrm{kV}$, which is about $0.5 \%$ relative PFN voltage variation (for a nominal PFN voltage of $30 \mathrm{kV}$ ) for $0.1 \%$ energy variation. This empirical relationship can be written as

$$
\frac{\delta E}{E} \sim 0.0472 \delta V^{2}
$$

which means that energy variations due to phase (via a PFN voltage variation) are about a factor of 5 less than direct $\mathrm{PFN}$-induced output power variations for a nominal PFN voltage of $30 \mathrm{kV}$. This is because the beam is accelerated on crest in the linac accelerating structures which means that the energy variation depends only to second order on the phase for small phase variations induced by the PFN.

\section{Model of Klystron Operation to Explain the Empirical Results}

In the last section we found that phase variations due to PFN voltage variations are much less important in terms of beam stability than the effect of directly lowering the output power of each klystron when the PFN voltage is varied. In this section a realistic model of klystron operation is presented that accounts for the empirical result expressed by Eq. (9).

The first result which is simply quoted here is the relationship between the voltage and current in a klystron tube amplifier given by

$$
I=K V^{n}
$$

which is the Langmuir-Child law when $n=3 / 2$ (4). To be clear, Eq. (12) is written in terms of the PFN voltage and in most cases is written in terms of the actual klystron tube voltage. Since the PFN and tube voltages simply differ by a scale factor (the transformer between the PFN and tube is a 15:1 step up) one can consider the constant $K$ to take this into account. The power output of the klystron is given by the relation

$$
P=\epsilon I V,
$$

where $V$ is the PFN voltage, $I$ is the klystron tube current, and $\epsilon$ is an efficiency factor that takes into account the klystron efficiency as well as the scale factor relating the tube voltage to the PFN voltage. 
Using Eqs. (1), (12), and (13) the expression for the energy gain for a given linac sector can be expressed as,

$$
E_{g}=\sqrt{\mathrm{R} \epsilon} V^{\frac{n+1}{2}},
$$

and the variation $\delta E_{g}$ in terms of the variation $\delta V$ is given by

$$
\delta E_{g}=\left(\frac{n+1}{2}\right) E_{g} \frac{\delta V}{V} .
$$

Using Eqs. (8) and (15), the beam energy variation for a given PFN voltage variation, assuming the PFN voltage varies the same way for each klystron, is

$$
\frac{\delta E}{E}=\left(\frac{n+1}{2}\right)\left(\frac{E-E_{\circ}}{E}\right) \frac{\delta V}{V}
$$

For $n=3 / 2$ and using the fact that the energy term is approximately unity, the energy variation is

$$
\frac{\delta E}{E}=\frac{5}{4} \frac{\delta V}{V}
$$

which is within about $20 \%$ the empirical result given by Eq. (9). This model as well as the emprical results show that if the PFN voltage varies by more than a given relative amount, the beam energy injected into the PAR will vary by the same relative amount.

\section{Conclusion}

Measurements and analysis of the positron linac accelerating structure energy gain and klystron gain were made and used to estimate the amount of allowable PFN voltage variation for a given upper bound on the energy variation of the beam injected into the PAR. The exponential model used in the klystron power gain data analysis is only an approximation to real klystron operation. Nevertheless, using this model, the klystron output power was very well fit. Using this approximate model along with measurements of the linac accelerating structure shunt impedance, an empirical expression was found relating the beam energy variation to a PFN voltage variation. The empirical relationship was explained using a more realistic model of klystron operation which relates the klystron tube current to voltage using the Langmuir-Child law (4). Finally; the positron beam energy variation due to $\mathrm{PFN}$-induced phase fluctuation was investigated and found to be less important than direct amplitude variations because the beam is accelerated on crest in the accelerating structures. This means that $\mathrm{PFN}$-induced phase variations only affect the beam energy to second order for small PFN voltage changes. 


\section{REFERENCES}

1. M. Borland, private communication.

2. Z. D. Farkas, H. A. Hogg, G. A. Loew, and P. B. Wilson, "SLED: A Method of Doubling SLAC's Energy," SLAC-PUB-1453, June 1974.

3. M. Borland, "A Self-Describing File Protocol for Simulation Integration and Shared Post-Processing," Proceedings of the 1995 Particle Accelerator Conference, Dallas, TX, 2184-2186 (1996).

4. M. Reiser, Theory and Design of Charged Particle Beams, John Wiley \& Sons, Inc., 46 (1994). 